\title{
Instability of the symmetric Couette-flow in a granular gas: hydrodynamic field profiles and transport
}

\author{
M. Sasvári ${ }^{(1,2)}$ J. Kertész ${ }^{(1)}$ and D.E. Wolf ${ }^{(2)}$ \\ (1) Department of Theoretical Physics, Technical University of Budapest, Budafoki út 8, H-1111 Hungary \\ (2) University Duisburg, D-45478 Duisburg, Germany
}

\begin{abstract}
We investigate the inelastic hard disk gas sheared by two parallel bumpy walls (Couette-flow). In our molecular dynamic simulations we found a sensitivity to the asymmetries of the initial condition of the particle places and velocities and an asymmetric stationary state, where the deviation from (anti)symmetric hydrodynamic fields is stronger as the normal restitution coefficient decreases. For the better understanding of this sensitivity we carried out a linear stability analysis of the former kinetic theoretical solution [Jenkins and Richman: J. Fluid. Mech. 171 (1986)] and found it to be unstable. The effect of this asymmetry on the self-diffusion coefficient is also discussed.
\end{abstract}

45.70M,45.70Q,51.10,51.20

\section{INTRODUCTION}

In the last decade the clustering instability of undriven granular gases was extensively researched. Since the first explanation of the instability 怔 investigation had been done with several methods including stability analysis of the hydrodynamic equations [2]3] and fluctuating hydrodynamics [4]. The examination of the influence of nonlinear coupling between hydrodynamic modes was also performed [5], and the long time behavior of the clustered state was with mode-coupling theories examined [6]. The evolution of vortex velocity patterns preceding the clustering instability is well understood [ [ ], and the minimal system size, where the instability appears, and its dependence on the restitution coefficient are revealed [5.:7].

A driven configuration, the uniformly sheared inelastic hard sphere gas (with periodic boundary conditions) shows also an instability and a resulting pattern formation. In [8] it was shown that this pattern formation was guided by an instability for the short times and a shearing caused convection for longer time-scales. The special importance of this system is the coupling of macroscopic and microscopic length-scales in sheared situations as discussed in 9911 .

A more realistic configuration is the Couette-flow, the shearing of the gas between two parallel walls moved in opposite directions. For this configuration with walls consisting of disks Jenkins and Richman derived boundary conditions for the momentum and heat transfer of the walls [12] proceeding from their kinetic theory for dense granular gases [13]. In subsequent years this theory was developed further [14] for non Maxwellian velocity distributions as in 12. In all of these papers the resulting hydrodynamic fields are symmetric (density and granular temperature) or antisymmetric (flow field) on the half-point between the two bounding walls. In view of the clustering instability it is of major interest whether this instability occurs in this configuration and, if so, what are its consequences in the Couette-flow. This paper is devoted to the investigation of these questions.

The paper is organized as follows: In the next Section (II) we present molecular dynamic simulations of this system where we found an interesting sensitivity to the initial condition of the simulation and asymmetric hydrodynamic fields contradicting the results in $\sqrt{12}$. Therefore we carried out the linear stability analysis (Sec. III) around the solution of Jenkins and Richman 12 and found it to be unstable against certain fluctuations. We discuss the effect of this instability on the diffusion coefficient in Sec. IV and close the paper with summary in Sec. V.

\section{SIMULATION RESULTS}

The system considered here consisted of $N$ identical, inelastic hard disks with mass $m=1$ and radius $r=1$ confined in a rectangular area of the size $L_{x} \times L_{y}$. This two-dimensional area is bounded by two parallel walls from two sides which also define the direction $x$ and are of the length $L_{x}$. The system is closed through periodic boundary conditions in the $x$ direction. The walls are $L_{y}$ distance apart ( $y$ direction). The origin of the coordinate system is placed in the middle of the simulated system what sets the center of the wall disks to $y= \pm L_{y} / 2$.

The disks interact through the inelastic rough hard sphere potential meaning instantaneous two particle collisions characterized by given ratios of the final and initial velocities in the normal and tangential direction as the normal and tangential restitution coefficients $e_{n}=-v_{n}^{f} / v_{n}^{i}$ and $e_{t}=v_{t}^{f} / v_{t}^{i}, f$ and $i$ meaning the final and initial velocities. 
In case of sliding contacts $e_{t}$ is replaced by the ratio of tangential and normal momentum transfer characterized by the friction coefficient $\mu$. As a driving force we move one of the walls with constant velocity $2 U$.

We investigated the system with the event-driven molecular dynamics method ideal for simulating instantaneous collision rules 15]. Characterizing the collisions we used $e_{t}=-0.3$ and $\mu=0.2$ and varied $e_{n}$ between 0.6 and 0.99. We consider the system sized $L_{x}=L_{y}=40$ and the wall velocity $2 U=10$ if not otherwise mentioned. The post-collision velocities and angular velocities as a function of the pre-collision ones are (for $m=1, r=1$ ),

$$
\begin{aligned}
\mathbf{v}_{i}^{\prime} & =\mathbf{v}_{i}-\frac{1+e_{n}}{2}\left(\left(\mathbf{v}_{i}-\mathbf{v}_{j}\right) \mathbf{r}_{n}\right) \mathbf{r}_{n}+\frac{j_{t}}{m} \mathbf{r}_{t} \\
\mathbf{v}_{j}^{\prime} & =\mathbf{v}_{j}+\frac{1+e_{n}}{2}\left(\left(\mathbf{v}_{i}-\mathbf{v}_{j}\right) \mathbf{r}_{n}\right) \mathbf{r}_{n}+\frac{j_{t}}{m} \mathbf{r}_{t} \\
\boldsymbol{\omega}_{i}^{\prime} & =\boldsymbol{\omega}_{i}-\frac{j_{t}}{I_{i}}\left(\mathbf{r}_{n} \times \mathbf{r}_{t}\right) \\
\boldsymbol{\omega}_{j}^{\prime} & =\boldsymbol{\omega}_{i}-\frac{j_{t}}{I_{j}}\left(\mathbf{r}_{n} \times \mathbf{r}_{t}\right)
\end{aligned}
$$

$\mathbf{r}_{n}$ being the normal vector of disk surfaces in collision $\mathbf{r}_{n}=\left(\mathbf{r}_{j}-\mathbf{r}_{i}\right) /\left|\mathbf{r}_{j}-\mathbf{r}_{i}\right|$ and $\mathbf{r}_{t}$ the tangential vector, the normal vector rotated with $\pi / 2$ in anti-clockwise direction. $I_{i}, I_{j}$ are the moments of inertia of the disks. The parameter $j_{t}$ depends on the type of the collision in tangential direction,

$$
\begin{array}{lll}
j_{t}=-\mu \frac{1+e_{n}}{2}\left(\mathbf{v}_{i}-\mathbf{v}_{j}\right) \mathbf{r}_{n} & \text { if } \quad \phi>\phi_{0} & \text { (sliding contact); } \\
j_{t}=\frac{e_{t}-1}{7} \mathbf{v}_{t} & \text { if } \quad \phi \leq \phi_{0} \quad \text { (sticking contact); }
\end{array}
$$

where $\mathbf{v}_{t}$ is the tangential velocity

$$
\mathbf{v}_{t}=\left(\mathbf{v}_{i}-\mathbf{v}_{j}\right) \mathbf{r}_{t}+r\left(\boldsymbol{\omega}_{j}+\boldsymbol{\omega}_{j}\right)
$$

and $\phi_{0}$ separates the sliding and sticking regions in the angle of incidence $\phi=\left|\mathbf{v}_{t}\right| /\left(\left(\mathbf{v}_{i}-\mathbf{v}_{j}\right) \mathbf{r}_{n}\right)$,

$$
\phi_{0}=\frac{7}{2} \mu \frac{1+e_{n}}{1-e_{t}} .
$$

To avoid inelastic collapse 16], the intrinsic numerical breakdown of the method, we stopped the simulations when the time interval between two subsequent collisions became smaller than the precision of the computations as proposed in [1]; but this stop occurred only for the used smallest restitution coefficient $e_{n}=0.6$ and for special initial conditions where most of the particles did not move, and the well-known chain-like arrangement of particles could evolve after the simulation started. For the experiments below we used particle numbers $N=100-240$ meaning area fraction $\nu \in[0.245,049]$. As test runs for larger systems we used $\left\{L_{x}=L_{y}=100, N=500,(\bar{\nu}=0.162)\right\}$ and $\left\{L_{x}=L_{y}=100, N=1000,(\bar{\nu}=0.324)\right\}$ parameter sets resulting in the same phenomenon.

Using several different starting configurations we noticed that there appears a dense region at one of the walls in the system which is more and more distinct with decreasing $e_{n}$ and not recognizable for $e_{n}$ near 1 , FIG 1 . Using randomly placed particles and an initial uniform velocity distribution with several mean values in the $x$ direction we observed that the initial value of the mean particle velocity determines the wall which is chosen for building up a clustered regime. If we define $v_{d}$ as

$$
v_{d}=\langle v\rangle-U
$$

we can characterize the final place as if $v_{d}>0$ the upper $y=L_{y} / 2$ wall is chosen, if $v_{d}<0$ the lower $y=-L_{y} / 2$ one, FIG 2. We used different initial conditions to test this finding [17]. With particles placed in a stripe in the middle of the system, organized on a lattice with initial velocity $U$, and using two of them shot against the two wall with initial velocities which were Galilei-symmetric with the situation of two walls moved with $\pm U$ we could maximize the time needed to develop the non-symmetric density field but it appeared also after this initial condition because numerical errors provide the needed fluctuations to drive the system in one of the steady states.

We did simulations for smooth disks $\left(e_{t}=1\right.$ and $\left.\mu=0\right)$ as well and found the same effect. Namely, if we chose $e_{n}$ to be small enough there is a distinct band of particles at one of the walls and for $e_{n}$ close to 1 this band does not evolve. The normal restitution below which we can speak about an asymmetric phase depends on the density as for higher densities it appears at higher $e_{n}$ values on the same system size $L_{y}$. It also depends on $L_{y}$ for fixed 
density as for larger $L_{y}$ the kinetic energy influx per particle decreases as it is proportionate with the length of the wall. The assumed critical restitution coefficient $e_{c}\left(\rho, L_{y}\right)$ does not depend on the driving velocity as $U$ is the only parameter which includes dimensionality of time and changing $U$ is a rescaling of the time unit. For the investigation of intermediate $e_{n}$ values for which the dense stripe is not so definite we measured density and velocity profiles and the granular temperature in the system and found the same effect now in the asymmetric nature of this functions FIG 3. One can also observe that with decreasing restitution the minimum of the granular temperature moves to one of the walls.

We found these results valid for a broad range of densities and for different system sizes. For low restitution there appeared a crystalline structure as compact layers near a wall. The fact that the simulations did not stop at such dense configuration implies that the disks moved around a fixed place and did not form a long enough connected line of particles in which inelastic collapse could occur [16].

Simulations with time step driven molecular dynamics using damped harmonic oscillator forces between the particles as a function of their overlap 15 - for achieving velocity independent restitution - show the same clustering effect at the walls. This suggests that this effect is closely related to the dissipative collisions and does not require instantaneous collision rules.

\section{STABILITY OF THE JENKINS-RICHMAN SOLUTION}

Our above findings contradict the kinetic theoretical calculation of Jenkins and Richman [12] and the improved versions of the problem [14] inasmuch as the cited results are always (anti)symmetrical profiles in the $y$ direction for the hydrodynamic fields of density, flow velocity and granular temperature. This contradiction raises the question of the stability of the (anti)symmetric solution. Moreover, it is plausible that in an elastic system the hydrodynamic profiles are symmetric, though this is not a steady state because of the ever rising temperature caused by viscous heating. Therefore it is of interest to see weather a phase transition occurs in the system, specifically, weather there exists a critical $e_{n}$ value or the (anti)symmetric solution becomes unstable at arbitrarily small inelasticity. Because at high densities the instability in question arises at normal restitution closer to 1 and kinetic theoretical calculations are more and more inadequate for increasing inelasticity it is reasonable to use kinetic theories for dense systems and we desist from the consideration of more sophisticated hydrodynamic equations for low density systems [18, 19]. Another reason for taking theories for dense gases into consideration is that the underlying assumptions are more valid as the ratio of the simulated system size to the mean free path is much larger in a dense gas. For this reasons we performed a linear stability analysis of the Jenkins-Richman solution. We now first recite the equations of Jenkins and Richman [13] and the boundary conditions and the solution of the problem [12]. Then we perform a linearization around this solution and calculate numerically the eigenvalues of the stability matrix.

\section{A. Jenkins-Richman Solution}

In this section we briefly describe the Jenkins and Richman 12] solution for a two-dimensional system of inelastic hard disks driven by two parallel bumpy walls. The hydrodynamic equations for the density $\rho$, flow velocity $\mathbf{u}$ and fluctuation energy $T$ for smooth disks without external forces are according to [13]:

$$
\begin{aligned}
\dot{\rho} & =-\rho \nabla \cdot \mathbf{u}, \\
\rho \dot{\mathbf{u}} & =-\nabla \cdot \mathbf{P}, \\
\rho \dot{T} & =-\nabla \cdot \mathbf{Q}-\operatorname{Tr}(\mathbf{P} \cdot \nabla \mathbf{u})-\gamma .
\end{aligned}
$$

Here the dissipation rate $\gamma$ and heat transport coefficient $\kappa$ are of the form,

$$
\begin{gathered}
\gamma=\frac{4\left(1-e_{n}\right) \kappa T}{\sigma^{2}} \\
\kappa=\frac{2 \rho \sigma \nu g_{0} T^{\frac{1}{2}}}{\pi^{\frac{1}{2}}}
\end{gathered}
$$

where $g_{0}$ is the Enskog correction term accounting for excluded volume effects as a function of the packing fraction $\nu$ :

$$
g_{0}=\frac{16-7 \nu}{16(1-v)^{2}}
$$


and $\sigma=2 r$ is the disk diameter. The constitutive relations for the energy flux $\mathbf{Q}$ and pressure tensor $\mathbf{P}$ are,

$$
\begin{aligned}
& \mathbf{Q}=-\kappa \nabla T \\
& \mathbf{P}=\left(2 \rho \nu g_{0} T-\frac{1}{2} \kappa \operatorname{Tr}(\mathbf{D}) \mathbf{I}-\kappa \mathbf{D} .\right.
\end{aligned}
$$

From the boundary geometry and the assumption of a Maxwellian velocity distribution the momentum and fluctuation energy supplied by the wall in unit time and length are determinable [12]. This needs an expansion in $\epsilon=\sigma / L_{y}$ and assumptions on the dependence of given ratios of the hydrodynamic fields on $\epsilon$ (see [12]), what narrows the validity of the theory. The supplied momentum obtained that way is

$$
M_{\alpha}=\frac{1}{2} \rho \chi\left(1+e_{n}\right) T\left(n_{\alpha}+\left(\frac{2}{\pi}\right)^{\frac{1}{2}} \frac{v_{\alpha}}{T^{\frac{1}{2}}}\left(\frac{\theta}{\sin \theta}-\cos \theta\right)+\left(\frac{2}{\pi}\right)^{\frac{1}{2}} \frac{\sigma}{T^{\frac{1}{2}}} u_{\gamma, \beta} I_{\alpha \beta \gamma}\right)
$$

with

$$
I_{\alpha \beta \gamma}=\left(\frac{2}{3} \sin ^{2} \theta-2\right) n_{\alpha} n_{\beta} n_{\gamma}-\frac{2}{3} \sin ^{2} \theta\left(n_{\alpha} t_{\beta} t_{\gamma}+n_{\beta} t_{\alpha} t_{\gamma}+n_{\gamma} t_{\alpha} t_{\beta}\right)
$$

and the supplied fluctuation energy is

$$
D=\left(\frac{2}{\pi}\right)^{\frac{1}{2}} \frac{\rho \chi\left(1-e_{n}\right) T^{\frac{3}{2}}}{\sin \theta}
$$

where $\mathbf{n}$ and $\mathbf{t}$ are the normal and tangential vectors of the wall respectively and $\theta$ characterizes the bumpyness of the wall. For wall disks with the same disk diameter as of the gas particles it equals [12]:

$$
\sin \theta=\frac{\sigma}{d}
$$

where $d$ is the distance separating the centers of two wall disks. For the wall geometry of our considered system $d=2 \sigma$ and therefore $\sin \theta=1 / 2$ and $\theta=\pi / 6$. In (16,18) $\chi$ accounts for static correlation effects in collisions at the boundary and $v_{\alpha}, \alpha=x, y$ is the slip velocity, the difference between the velocity of the wall and the flow $\mathbf{v}=\mathbf{U}-\mathbf{u}$. From the equality of momentum and energy transfer of the wall for unit time and unit length the boundary conditions are:

$$
\begin{aligned}
\mathbf{M} & =\mathbf{P} \cdot \mathbf{n}, \\
\mathbf{M} \cdot \mathbf{v}-D & =\mathbf{Q} \cdot \mathbf{n} .
\end{aligned}
$$

The solution can be calculated from these equations except one parameter - the value of solid fraction at the boundaries (for fixed number of particles) - which must be iterated to get the given density in the system what we did numerically. Thus the form of the hydrodynamic fields solving the boundary value problem is, with $T(y)=w(y)^{2}$,

$$
\begin{aligned}
& w(y)=\frac{\lambda(U-v) N \sigma}{2 \pi^{\frac{1}{2}} \sinh (\lambda / 2) S L} \cosh \left(\frac{\lambda y}{L}\right), \\
& u(y)=\frac{U-v}{\sinh (\lambda / 2)} \sinh \left(\frac{\lambda y}{L}\right) .
\end{aligned}
$$

with $S$ and $N$ being the shear stress and pressure respectively and $\lambda$ must be solved from [12],

$$
\frac{\lambda}{2} \tanh \left(\frac{\lambda}{2}\right)=\frac{\left(1-e_{n}\right) L \theta}{2 \sqrt{2} \sigma \sin \theta}\left(\frac{\sin \theta\left[1-(4 \sqrt{2 \sigma} / 3 \sigma) \sin ^{2} \theta\right]}{\theta(\theta / \sin \theta-\cos \theta)}-1\right) .
$$

The density, calculated from

$$
\chi=2 \nu g_{0}
$$

must fulfill the condition that its integral on the whole system gives the prepared particle number. As mentioned, in that condition the only free parameter remains the density at the boundaries what must be calculated iteratively. 


\section{B. Stability Analysis}

Around the above solution of the boundary value problem we performed a linear stability analysis with the perturbed quantities,

$$
\begin{aligned}
\rho^{\prime} & =\rho+\delta \rho \\
\mathbf{u}^{\prime} & =\mathbf{u}+\delta \mathbf{u} \\
T^{\prime} & =T+\delta T .
\end{aligned}
$$

We split the velocity perturbation in the $x$ and $y$ direction and use plane waves for the perturbations,

$$
\begin{aligned}
\delta \rho & =\delta \rho_{\mathbf{k}} \exp (-i \mathbf{k} \cdot \mathbf{r}) \\
\left(\begin{array}{c}
\delta u \\
\delta w
\end{array}\right) & =\left(\begin{array}{c}
\delta u_{\mathbf{k}} \\
\delta w_{\mathbf{k}}
\end{array}\right) \exp (-i \mathbf{k} \cdot \mathbf{r}) \\
\delta T & =\delta T_{\mathbf{k}} \exp (-i \mathbf{k} \cdot \mathbf{r}) .
\end{aligned}
$$

With this choice the resulting equations have the form

$$
\left(\begin{array}{c}
\partial_{t} \delta \rho_{\mathbf{k}} \\
\partial_{t} \delta u_{\mathbf{k}} \\
\partial_{t} \delta w_{\mathbf{k}} \\
\partial_{t} \delta T_{\mathbf{k}}
\end{array}\right)=-\mathbf{A}\left(\begin{array}{c}
\delta \rho_{\mathbf{k}} \\
\delta u_{\mathbf{k}} \\
\delta w_{\mathbf{k}} \\
\delta T_{\mathbf{k}}
\end{array}\right)
$$

where $\mathbf{A}$ is the stability matrix with the elements given in Appendix $\mathrm{A}$.

With the help of MAPLE V Release 5 we analyzed the eigenvalues of the stability matrix $\mathbf{A}$ for different densities, restitution coefficients, wave numbers and sites. On FIG 4-7 we present results for some of the parameter sets which are representative for the different situations. With the choice of the negative sign in (31) a negative eigenvalue means an unstable solution. All of the shown figures feature at least one eigenvalue which is negative in the small wave number region. This signalizes the unstable fluctuation. The upper zero point of this eigenvalue nears to the zero wave number as we increase the restitution coefficient and becomes smaller than $2 \pi / L$, the smallest possible wave number in the system, at a certain value of $e_{n}$ what depends on the other parameters. However, this value is very close to 1 what suggests that the symmetrical solution is always unstable linearly and nonlinear effects can move this transition point to higher inelasticities. We analyzed the most unstable direction of the solution also as we plotted the unstable eigenvalue for a given amplitude of the wave number and for the whole angle measured from the direction of the mean flow in anti-clockwise direction as seen in FIG 8,9. According to the figures the most unstable directions are close to the $\pm \pi / 4$ angles which are the same angles found to be the governing unstable directions in linear order for the pattern formation by Tan and Goldhirsch for the uniformly sheared granular gas [8].

\section{SELF-DIFFUSION COEFFICIENT AND CLUSTERED FLOWS}

In this section we investigate the crossover to asymmetric stationary states of the system and try to show the effect of the instability discussed above on the transport coefficients. For this reason we measured the mean square displacement of the particles,

$$
r_{x}^{2}=\left\langle\left(x(t)-\left\langle v_{x}\right\rangle t-x(0)\right)^{2}\right\rangle, \quad r_{y}^{2}=\left\langle(y(t)-y(0))^{2}\right\rangle
$$

and velocity correlation functions,

$$
c_{x}=\left\langle\left(v_{x}(t)-\left\langle v_{x}\right\rangle\right)\left(v_{x}(0)-\left\langle v_{x}\right\rangle\right)\right\rangle, \quad c_{y}=\left\langle v_{y}(t) v_{y}(0)\right\rangle
$$

in the system for stream-wise and perpendicular directions. In (32) and (33) the brackets \langle\rangle mean space and ensemble averages over the whole system. $\left\langle v_{x}\right\rangle$ was calculated from the measured average displacement of the particles averaged over several (50-100) runs and over particles. Ensemble averages were carried out the following way. For every parameter set we let relax the system in its steady state. Then measured granular temperature averaged over stripes of width $1=r / 2$ generating the $T(y)$ function. Before every measurement we perturbed the velocities of every particle with a uniformly distributed velocity the support of the distribution being $2 \sqrt{T(y)}$, where $y$ was the coordinate of 
the particle. After relaxation we carried out the measurement. The $\left\langle v_{x}\right\rangle$ average velocity was evaluated from a linear fit on the averaged $x$ displacement of the particles being the steepness of $x$ as a function of time.

From the mean square displacement for long times we could evaluate the diffusion coefficient of the particles. We fitted a power law with two fit parameters $D$ and $b$,

$$
f(t)=2 D t^{b}
$$

on the mean square displacement restricted to long times. If the value of $b$ was up to errors $( \pm 0.02)$ near 1 the system was assumed to be in a diffusive state. After that we took $b=1$ and fitted $f(t)$ again for $D$. The time the system evolves into this diffusive state depends on $e_{n}$ as suggested in [20] for the homogeneous cooling state, and also depends on the steady state configuration which is also $e_{n}$ dependent. This has several reasons. First, to achieve this diffusive behavior in $x$ direction the particles have to experience the characteristics of the system - finiteness and inhomogeneity of the hydrodynamic profiles - therefore have to travel between the to walls several times. As $e_{n}$ decreases and a dense cluster evolves at one wall this recognition takes more and more time. Also the form of the velocity correlation function shows increasing correlations with decreasing $e_{n}$ as expected. After a short $(t \approx 10)$ fast decay $c_{x}$ shows an exponential decay up to times $t \approx 100$ and changes to a slow decay for later times ( $\operatorname{small} e_{n}$ ) or is relaxed (large $e_{n}$ ) FIG 10. Both the short-time correlations and the characteristic time of the exponential increases as we decrease $e_{n}$, along with the earlier changeover to the slow decay if it is apparent.

The plot of the mean square displacement versus time diagram (FIG 11) shows a plateau value of the diffusion coefficient for larger values of $e_{n}$. Below $e_{c}=0.82 \mathrm{D}$ increases fast with decreasing $e_{n}$. We suggest that this value marks the transition point where the particles begin to prefer one of the walls and the appearing dilute regions allow for a greater mobility for the particles. Making use of the noticed exponential decay of the velocity autocorrelation $c_{x}$ we also estimated $D$ as the integral of a fitted exponential,

$$
D \approx A \tau \quad \text { if } \quad c_{x} \approx A e^{-t / \tau}
$$

knowing that it underestimates $D$ as $A e^{-t / \tau}$ is smaller than $c_{x}$ for very short and for long times, but it gives nearly the same result as the long time behavior of $r_{x}^{2}$ above the transition point and shows a smaller increase below it (FIG 11). This breakup comes from the fact that under the transition point there appears a slow decay in the velocity correlation function after the exponential decay used to approximate it. The short-time exponential decay characterizes only the dilute part of the system. The slow decay thereafter comes from the averaged effects of the exigous diffusion in the dense region and from the influence of particles entrapped or emitted by the dense region from or to the dilute one. In absence of the asymmetric dense state above the transition point $e_{c}$ only the exponential relaxation appears.

The buildup of correlations is also a cause for increasing times needed to reach the diffusive state thus increasing simulation time what restricted us to smaller system sizes for dense material as it did not allow fast computations. Therefore the investigation of the size and density dependence of the transition point is left for later studies.

The retrievable information from the mean square displacement in $y$ direction is bounded as the system itself namely it goes to a plateau value according to $L_{y}$. This finiteness influences velocity correlations also. $c_{y}$ shows only shorttime fast decaying correlations (and there appear anti-correlations for small $e_{n}$.) With the methods discussed above we do not noticed any mark of the transition (only a small sign as presented in [21] for time-step driven simulations).

For more definite insights in the behavior of the system we carried out measurements specific to the configuration of the stationary states. We divided the system into stripes parallel to the walls and restricted the averages in (33) on particles starting from these stripes,

$$
c_{x}^{i}=\left\langle\left(v_{x}(t)-\bar{v}_{x}^{i}(t)\right)\left(v_{x}(0)-\bar{v}_{x}^{i}(0)\right)\right\rangle_{i}, \quad c_{y}^{i}=\left\langle v_{y}(t) v_{y}(0)\right\rangle_{i}
$$

where $\left\langle>_{i}\right.$ denotes averaging over particles being in stripe $i$ at $t=0$ and the ensemble average described above. The velocity $\bar{v}_{x}^{i}(t)$ is the average velocity of particles starting from stripe $i$ at time $t$. This velocity correlation function characterizes the stripe more and more as the difficulty to leave the stripe increases which is the case in the dense layer evolving at the wall. The fact that the velocity correlation function $c_{x}^{i}$ does not show a negative minimum at short times (FIG.10) - which would be a sign of caging effects - suggests, that the particles essentially comoving with the wall move barely in the $x$ direction compared to one another. Instead because of the sheared situation they are driven by diagonal collisions which dominate the motion and smooth out $c_{x}$. There is a negative minimum in $c_{y}^{0}$ (0 indexing the stripe nearest to the wall at the cluster) but this includes the effect of the collisions with the wall particles which give a negative contribution as also seen on the velocity correlations in the stripe at the opposite wall. This appears also at restitution coefficients well above the transition. 


\section{DISCUSSION}

In this paper we considered a granular gas sheared by two bumpy walls. By means of molecular dynamics simulations we discovered that the system shows a spontaneous symmetry breaking as it evolves to a stationary state which is asymmetric in the hydrodynamic fields with respect to the centerline of the system. The location where this density maximum (temperature minimum) is shifted to from the symmetry axes is predetermined by the initial velocity distribution as the system chooses the wall according to this distribution as discussed in Sec. I1. During the simulations, measuring the mean $y$ coordinate of the particles, we did not observe any switch between the possible two states where the asymmetry was explicit. We found in a linear stability analysis that the symmetrical solution proposed by Jenkins and Richman [12] is unstable. In analyzing the solution [12] one finds that the description of the asymmetric profiles would need higher order equations in $\epsilon$ as in [12]. We do not consider this analysis as a strict result quantitatively. According to [22 the equations of Jenkins and Richman are not suitable for linear stability analysis because they neglect the time dependence of the mean free path. But the equations with the corrected constitutive relations [10] have the same dependence on the hydrodynamic field and differ in the $e_{n}$ dependence only. We assume that this correction terms cannot stabilize the system but can at most increase the $e_{n}$ value under which the solution becomes unstable.

We found a connection to the unstable directions observed in uniformly sheared granular gases [8]. That suggests that not uniformly sheared systems possess the same instability but in the Couette-case the pattern formation is repressed by the boundary conditions. However, the geometry of the boundary conditions (walls) plays a major role in stabilizing the flow fields leading to a stationary state. In the boundary conditions of Jenkins and Richman [12] only static correlations are considered and dynamic correlations, the role of multiple and correlated re- collisions with the wall, are neglected. We measured the number of collision sequences which consist of collisions of a disk with two wall disks successively because such collisions provide disks leaving the wall in a steeper angle what can provide greater contribution to the pressure at lower granular temperature. In the asymmetric flow regime the number of such collisions is clearly higher at the preferred wall than at the other what supports our assumption. This is also implied by the fact, that in the final stationary state the minimum of the granular temperature is only at the wall for very high inelasticities or densities where we could observe closed layers of particles at the wall chosen. For moderate densities and restitution the temperature minimum remains in the bulk of the system, and the clustering process stops at a stable density profile. Such effect was described for a constrained homogeneous system in [5]. This boundary effects are one reason for the different results for the transition point obtained from the stability analysis or the measurement of the self-diffusion coefficient and velocity autocorrelation functions respectively. The found effect in the dependency of these functions on the normal restitution coefficient described in Sec. IV is well described as a consequence of the transition. However, the system size used in the simulations is too small to compare the two results. We suggest that for larger systems where the Jenkins-Richman theory is more valid we would obtain larger values for the transition point but the involved measurements leave this for later studies. As a final note we would like to mention that in systems with elastic collisions with the wall disks the flow with the same parameters remains symmetric.

The consequences of this paper are hard to verify in experiments because of the absence of gravity. However microgravity experiments for the same configuration are under way [23]. We suggest that the two-dimensional case considered here would be realizable with disks on an air table as this would exclude gravity effects and would not interfere with the behavior of the disks in the crucial horizontal direction. Periodic boundary conditions could be achieved with two similar stadion-like chains of disks as performed in 23].

\section{ACKNOWLEDGEMENTS}

M.S (J.K) wants to acknowledge the financial help of the DAAD (AvH Foundation). This work was partially supported by OTKA T029985 and OTKA T024004.

\section{APPENDIX A: THE LINEAR STABILITY MATRIX}

In the following we describe the matrix elements of the linear stability matrix. The notations include $\rho=\vartheta \nu$ where $\vartheta=\pi / 4$ for $\sigma=1, m=1$ and $\varphi=\partial_{y}\left(\nu g_{0}(\nu)\right)$.

$$
\begin{aligned}
& A_{11}=u i k_{x} ; \\
& A_{12}=\rho i k_{x} ; \\
& A_{13}=\rho i k_{y}+\partial_{y} \rho ;
\end{aligned}
$$




$$
\begin{aligned}
& A_{14}=0 ; \\
& A_{21}=2 T \varphi i k_{x}+\frac{2 \nu g_{0} T i k_{x}}{\rho}-\frac{\sigma}{\sqrt{\pi}} \partial_{y}^{2} u\left(\frac{\nu g_{0} \sqrt{T}}{\rho}+\sqrt{T} \varphi \vartheta\right)- \\
& -\frac{\sigma}{\sqrt{\pi}} \frac{\partial_{y} u}{\rho}\left[\varphi \sqrt{T}+\nu g_{0}\left(\frac{\partial_{y} T}{2 \sqrt{T}}+\sqrt{T} i k_{y}\right)+\partial_{y} \rho \sqrt{T} \varphi \vartheta\right]- \\
& -\frac{\sigma}{\sqrt{\pi}} \partial_{y} u \vartheta\left(\frac{\partial_{y} T \varphi}{2 \sqrt{T}}+\sqrt{T} \partial_{y} \varphi+\sqrt{T} \varphi i k_{y}\right) ; \\
& A_{22}=u i k_{x}+\frac{\sigma \nu g_{0} \sqrt{T}}{\sqrt{\pi}}\left[3 k_{x}^{2}+k_{y}^{2}-\left(\frac{\partial_{y} \rho}{\rho}+\frac{\partial_{y} T}{2 T}\right) i k_{y}\right]-\frac{\sigma}{\sqrt{\pi}} \varphi \sqrt{T} i k_{y} ; \\
& A_{23}=\partial_{y} u+\frac{\sigma \nu g_{0} \sqrt{T}}{\sqrt{\pi}}\left[2 k_{x} k_{y}-\left(\frac{\partial_{y} \rho}{\rho}+\frac{\partial_{y} T}{2 T}\right) i k_{x}\right]-\frac{\sigma}{\sqrt{\pi}} \varphi \sqrt{T} i k_{x} ; \\
& A_{24}=2 \nu g_{0} i k_{x}-\frac{\sigma \nu g_{0} \partial_{y}^{2} u}{2 \sqrt{T \pi}}--\frac{\sigma \partial_{y}^{2} u}{2 \sqrt{T \pi}}\left[\varphi+\nu g_{0}\left(\frac{\partial_{y} \rho}{\rho}-\frac{\partial_{y} T}{2 T}+i k_{y}\right)\right] \text {; } \\
& A_{31}=2\left(\frac{\partial_{y} \rho T \varphi}{\rho}+\partial_{y} T \varphi+T \partial_{y} \varphi\right) \vartheta+2 T \varphi \vartheta i k_{y}+\frac{2 \varphi T}{\rho} ; \\
& +2 \frac{\nu g_{0}}{\rho}\left(\partial_{y} T+T i k_{y}\right)-\frac{\sigma \partial_{y} u}{\sqrt{\pi}}\left(\frac{\nu g_{0} \sqrt{T} i k_{x}}{\rho}+\sqrt{T} \varphi i k_{x} \vartheta\right) ; \\
& A_{32}=\frac{\sigma \nu g_{0} \sqrt{T}}{\sqrt{\pi}}\left[2 k_{x} k_{y}-\left(\frac{\partial_{y} \rho}{\rho}+\frac{\partial_{y} T}{2 T}\right) i k_{x}\right]-\frac{\sigma}{\sqrt{\pi}} \varphi \sqrt{T} i k_{x} ; \\
& A_{33}=u i k_{x}+\frac{\sigma \nu g_{0} \sqrt{T}}{\sqrt{\pi}}\left[k_{x}^{2}+3 k_{y}^{2}-\left(\frac{\partial_{y} \rho}{\rho}+\frac{\partial_{y} T}{2 T}\right) 3 i k_{y}\right]-\frac{\sigma}{\sqrt{\pi}} \varphi \sqrt{T} 3 i k_{y} ; \\
& A_{34}=2 \nu g_{0}\left(\frac{\partial_{y} \rho}{\rho}+i k_{y}\right)+2 \varphi-\frac{\sigma \nu g_{0} \partial_{y} u i k_{x}}{2 \sqrt{t \pi}} ; \\
& A_{41}=\left(\frac{8\left(1-e_{n}\right)}{\sigma \sqrt{\pi}} T^{\frac{3}{2}}-\frac{2 \sigma \sqrt{T} \partial_{y}^{2} T}{\sqrt{\pi}}\right)\left(\frac{\nu g_{0}}{\rho}+\varphi \vartheta\right) \\
& -\frac{2 \sigma \partial_{y} T}{\sqrt{\pi} \rho}\left[\varphi \sqrt{T}+\nu g_{0}\left(\frac{\partial_{y} T}{2 \sqrt{T}}+\sqrt{T} i k_{y}\right)+\varphi \partial_{y} \rho \sqrt{T} \vartheta\right] \\
& -\frac{2 \sigma \partial_{y} T \vartheta}{\sqrt{\pi}}\left[\partial_{\varphi} \sqrt{T}+\frac{\varphi \partial_{y} T}{2 \sqrt{T}}+\varphi \sqrt{T} i k_{y}\right]-\frac{\sigma \sqrt{T}}{\sqrt{\pi}}\left(\partial_{u}\right)^{2}\left(\frac{\nu g_{0}}{\rho}+\varphi \vartheta\right) ; \\
& A_{42}=2 \nu g_{0} T i k_{x}-\frac{2 \sigma}{\sqrt{\pi}} \nu g_{0} \sqrt{T} \partial_{y} u i k_{y} \\
& A_{43}=\partial_{y} T+2 \nu g_{0} T i k_{y}-\frac{2 \sigma}{\sqrt{\pi}} \nu g_{0} \sqrt{T} \partial_{y} u i k_{x} ; \\
& A_{44}=u i k_{x}+\frac{12\left(1-e_{n}\right) \nu g_{0} \sqrt{T}}{\sigma \sqrt{\pi}} \\
& +\frac{2 \sigma \nu g_{0} \sqrt{T}}{\sqrt{\pi}}\left[k_{x}^{2}+k_{y}^{2}-\left(\frac{\partial_{y} \rho}{\rho}+\frac{\partial_{y} T}{2 T}\right) i k_{y}\right]-\frac{2 \sigma}{\sqrt{\pi}} \varphi \sqrt{T} i k_{y}-\frac{\sigma \nu g_{0}}{\sqrt{T \pi}} \partial_{y}^{2} T \\
& -\frac{\sigma}{\sqrt{T \pi}} \partial_{y} T\left[\varphi+\nu g_{0}\left(\frac{\partial_{y} \rho}{\rho}-\frac{\partial_{y} T}{2 T}\right)+\nu g_{0} i k_{y}\right]-\frac{\sigma \nu g_{0}}{2 \sqrt{T \pi}}\left(\partial_{y} u\right)^{2} \text {; }
\end{aligned}
$$

[1] I. Goldhirsch, G. Zanetti, Phys. Rev. Lett. 70, 1619 (1993).

[2] S. McNamara, Phys of Fluids A 5, 3056 (1993). 
[3] J.J. Brey, M.J. Ruiz-Montero, F. Moreno, Physics of Fluids 10, 2976 (1998).

[4] T.P.C. van Noije, M.H. Ernst, Phys. Rev. E 61, 1765 (2000).

[5] J.J. Brey, R.J. Ruiz-Montero, D. Cubero, Phys. Rev. E 60, 3150 (1999).

[6] R. Brito, M.H. Ernst: Europhys. Lett. 43 (5) pp. 497-502 (1998);

[7] J.T. Jenkins, J. Appl. Mech. 59, 120 (1992).

[8] M-L. Tan, I. Goldhirsch, Phys. Fluids 9, (4) 856 (1997).

[9] N. Sela, I. Goldhirsch, S.H. Noskovitz, Phy. Fluids 8, (9) 2337 (1996).

[10] N. Sela I. Goldhirsch, J. Fluid. Mech. 361, 41 (1998).

[11] M-L. Tan, I. Goldhirsch, Phys. Rev. Lett. 81, 3022 (1998).

[12] J.T. Jenkins M.W. Richman, J. Fluid. Mech. 171, 53 (1986).

[13] J.T. Jenkins M.W. Richman, Phys. Fluids 28, 3485 (1985).

[14] M.W. Richman C.S. Chou, J. of Appl. Math. and Phys. (ZAMP) 39, 885 (1988).

[15] D.E. Wolf, Modeling and Computer Simulation of Granular Media in Computational Physics eds. K. H. Hoffmann and M. Schreiber (Springer, Heidelberg, 1996).

[16] McNamara, W.R. Young, Phys. Rev. E 50, R28 (1994); Phys. Rev. E 53, 5089 (1996).

[17] M. Sasvári, J. Kertész, D.E. Wolf, in Traffic and Granular Flow '97, eds. M. Schreckenberg, D. E. Wolf, Springer (1998).

[18] J. J. Brey, J. W. Dufty, C. S. Kim, A. Santos, Phys. Rev. E 584638 (1998)

[19] V. Garzó, J. W. Dufty, Phys. Rev. E 595895 (1999).

[20] J. J. Brey, M. J. Ruiz-Montero, D. Cubero, R. Garcia-Rojo, Phys. Fluids 12, 876 (2000).

[21] M. Sasvári, J. Kertész, D. E. Wolf, Anomalous Diffusion, Lecture Notes in Physics 519, p349-357 (1999).

[22] I. Goldhirsch, Chaos 9, 659 (1999).

[23] M. Y. Louge, J. T. Jenkins: Poster at the Granular Gases Workshop, Bad Honnef, 1999;

\section{FIGURE CAPTIONS}

FIGURE 1: Snapshot configurations in the steady state for different restitution coefficients: $(1 \mathrm{a}) e_{n}=0.7,(1 \mathrm{~b})$ $e_{n}=0.8,(1 \mathrm{c}) e_{n}=0.9$ Lines from centers of particles indicate the direction and magnitude of its velocity.

FIGURE 2: Snapshot configurations in the steady state for different initial velocity distributions $\left(e_{n}=0.7\right)$. 2a: $v_{d}<0,2 \mathrm{~b}: v_{d}>0$.

FIGURE 3: Granular temperature in the $x$ and $y$ direction as a function of $y$ (in units of disk radius $r=1$ ) across the system for $e_{n}=0.9$. Values are averages over stripes of width 4 parallel to the walls with diameter 4 for a system of size $L_{y}=40$.

FIGURE 4: Eigenvalues of the stability matrix for $e_{n}=0.9, y=0$, average packing fraction $\bar{\nu}=0.486$, and $\mathbf{k}_{\alpha}$ points in the direction $\alpha=\pi / 4$ measured anti-clockwise from the mean flow direction.

FIGURE 5: Eigenvalues of the stability matrix for $e_{n}=0.95, y=0, k_{x}=2 \pi / L$ and average packing fraction $\bar{\nu}=0.486$ as a function of $k_{y} L / \pi$.

FIGURE 6: Eigenvalues of the stability matrix for $e_{n}=0.997, y=0$, average packing fraction $\bar{\nu}=0.486$, and $\mathbf{k}_{\alpha}$ points in the direction $\alpha=\pi / 4$ measured anti-clockwise from the mean flow direction.

FIGURE 7: Eigenvalues of the stability matrix for $e_{n}=0.999, y=0$, average packing fraction $\bar{\nu}=0.486$, and $\mathbf{k}_{\alpha}$ points in the direction $\alpha=\pi / 4$ measured anti-clockwise from the mean flow direction.

FIGURE 8: Angle dependence of the real part of the eigenvalues for $e_{n}=0.95, y=0$ and $\bar{\nu}=0.486$. The eigenvalue is plotted as a function of the angle measured from the flow direction $k_{x}=(2 \pi / L) \cos (\phi)$ and $k_{y}=(2 \pi / L) \sin (\phi)$.

FIGURE 9: Angle dependence of the real part of the eigenvalue for $e_{n}=0.997, y=0$ and $\bar{\nu}=0.486$. The eigenvalue is plotted as a function of the angle measured from the flow direction $k_{x}=(2 \pi / L) \cos (\phi)$ and $k_{y}=(2 \pi / L) \sin (\phi)$.

FIGURE 10: Velocity autocorrelation functions $c_{x}$ (in units of $U^{2}=25$ ) for a system with parameters $L_{x}=20$, $L_{y}=20, N=50,(\bar{\nu}=0.426)$. In order of increasing values for short times are $e_{n}=0.8,0.78,0.77,0.76$. Inset: Velocity autocorrelation functions $c_{x}^{i}$ (same units) for a system $L_{x}=80, L_{y}=20, N=200,(\bar{\nu}=0.426)$ and $e=0.76$. The system was divided in 5 stripes of width 4 . Correlation functions are shown only in the stripes at the walls. Solid line marks the function at the wall of the cluster, and dot-dashed line at the opposite wall. Time is measured in natural units $(r=1, U=5)$.

FIGURE 11: The self-diffusion coefficient as a function of the restitution coefficient $e_{n}$ for $L_{x}=L_{y}=20, N=50$, $(\bar{\nu}=0.426)$ measured in natural units. This runs were carried out moving both walls in opposite direction with $U= \pm 5$. Diamonds $(\diamond)$ marking values determined from the mean square displacement, and triangles $(\triangle)$ marking values determined from the velocity autocorrelation function. 
00000000000000000000

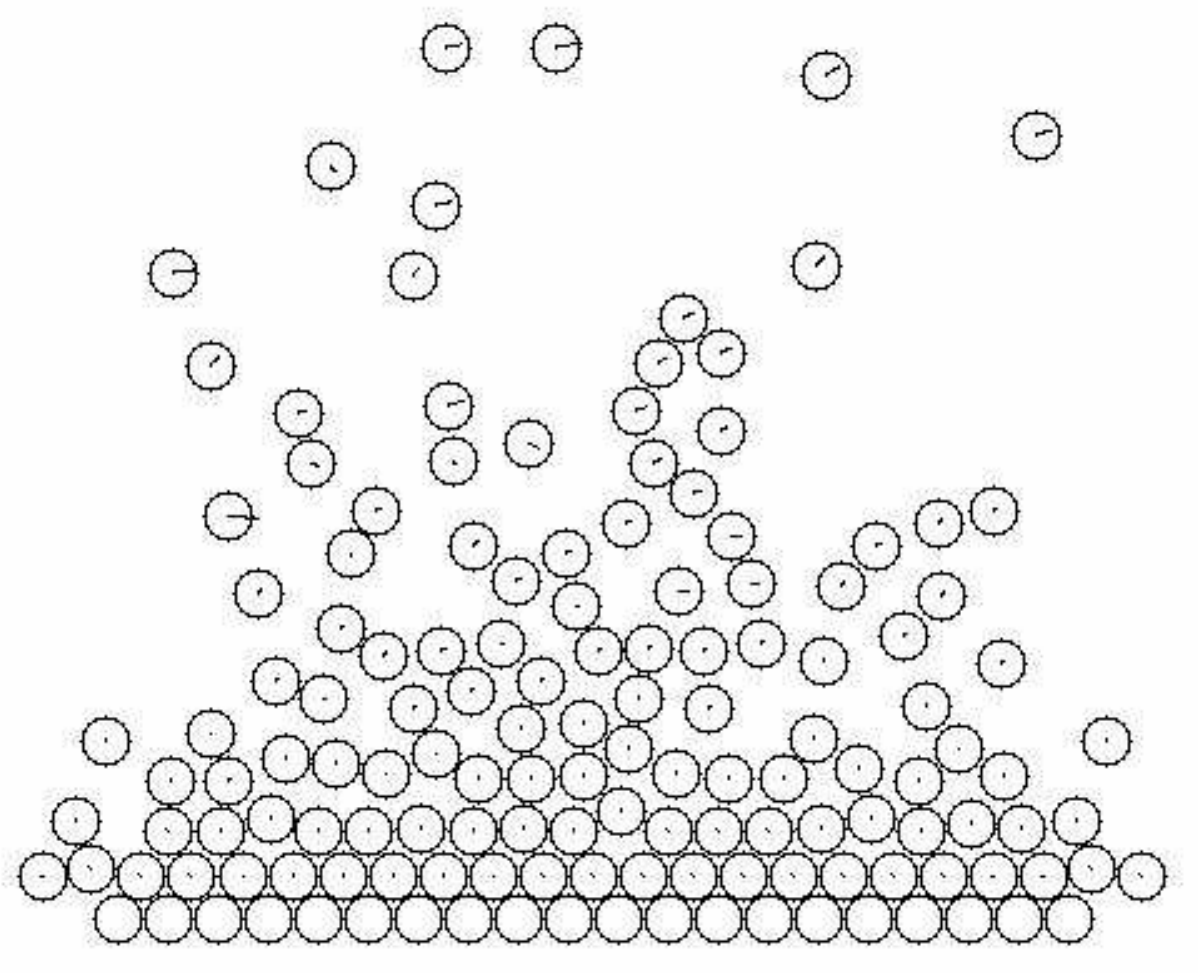

FIG. 1a 
00000000000000000000

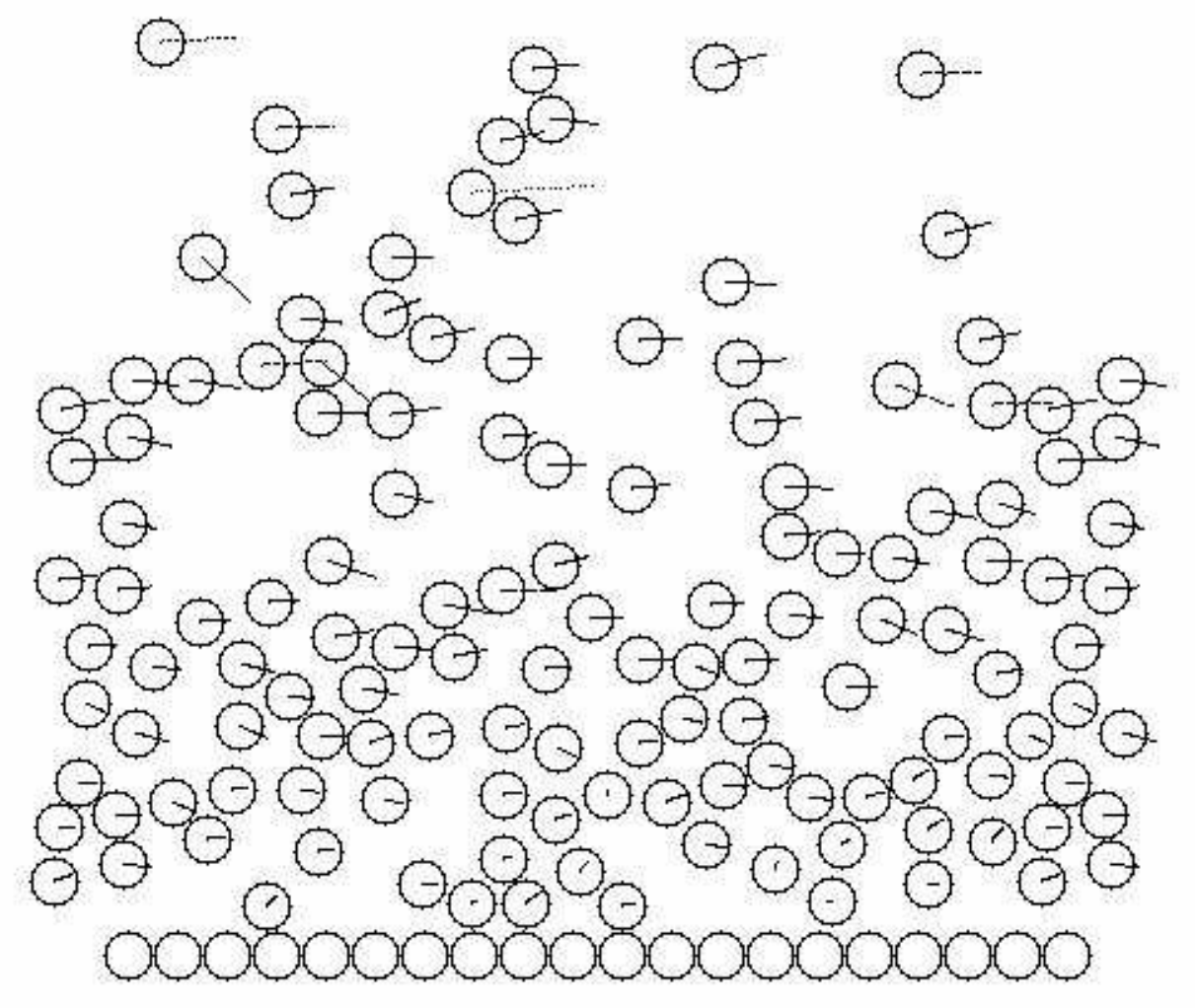

FIG. 1b 


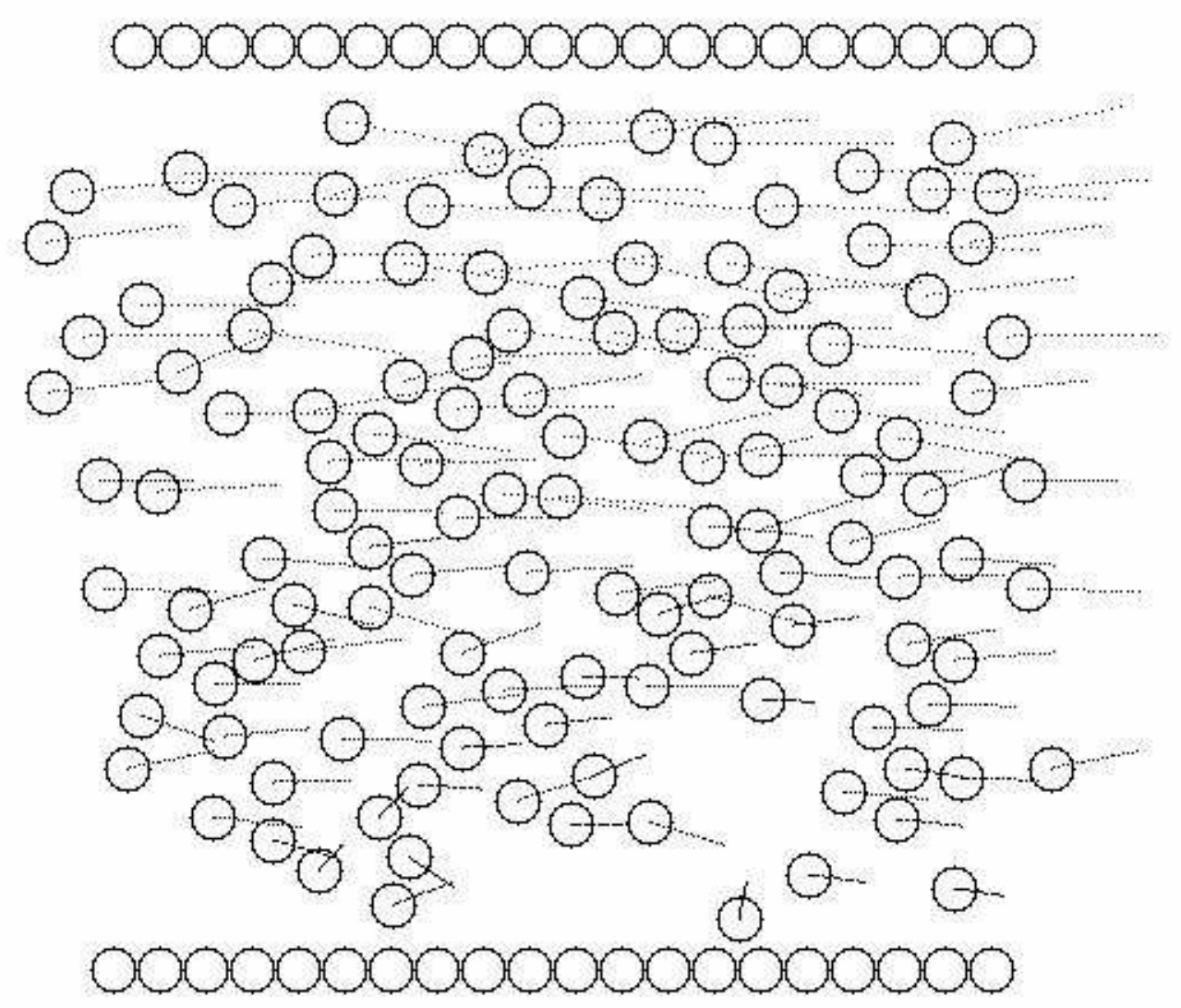

FIG. 1c 


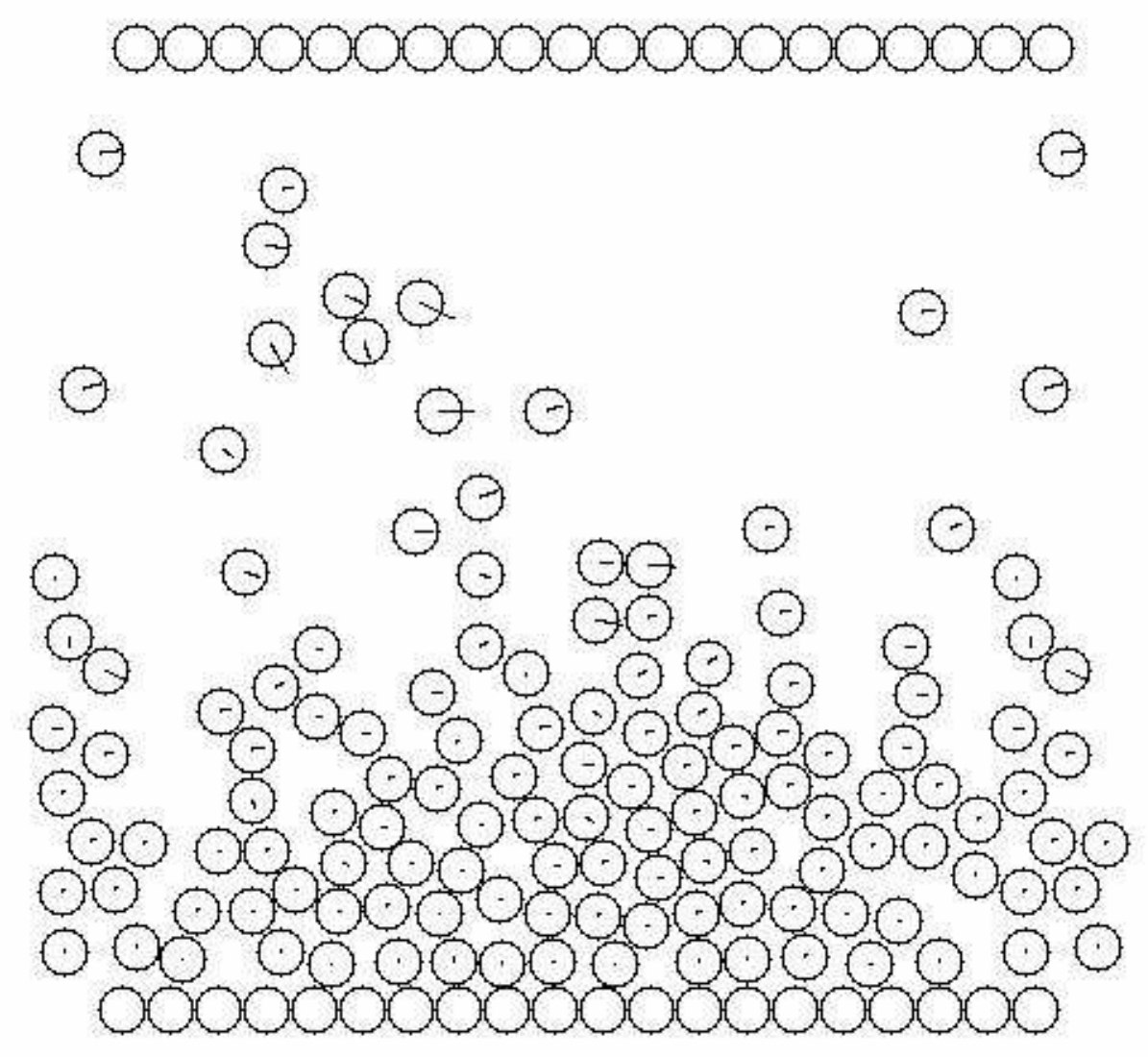

FIG. 2a 


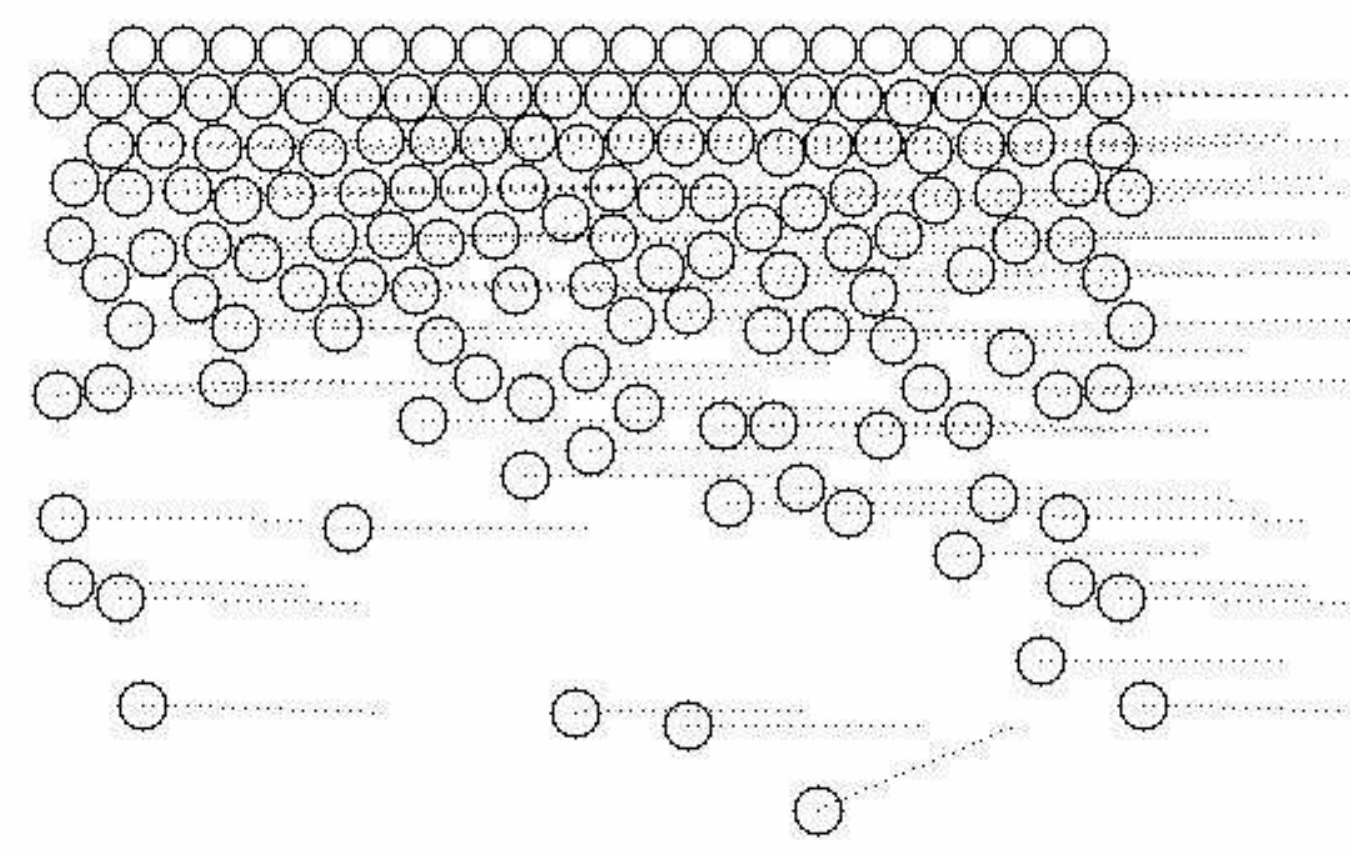

00000000000000000000

FIG. 2b 


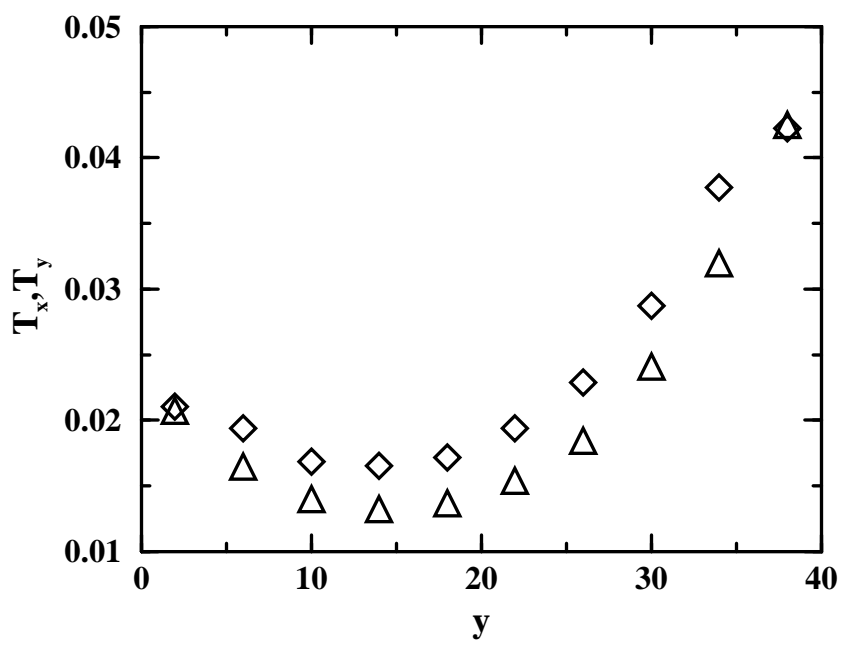

FIG. 3 


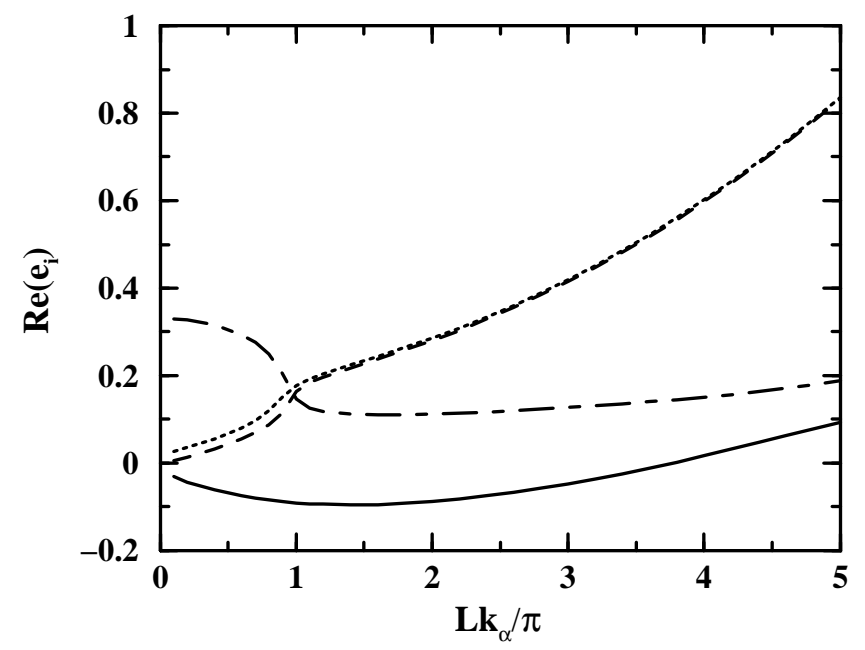

FIG. 4 


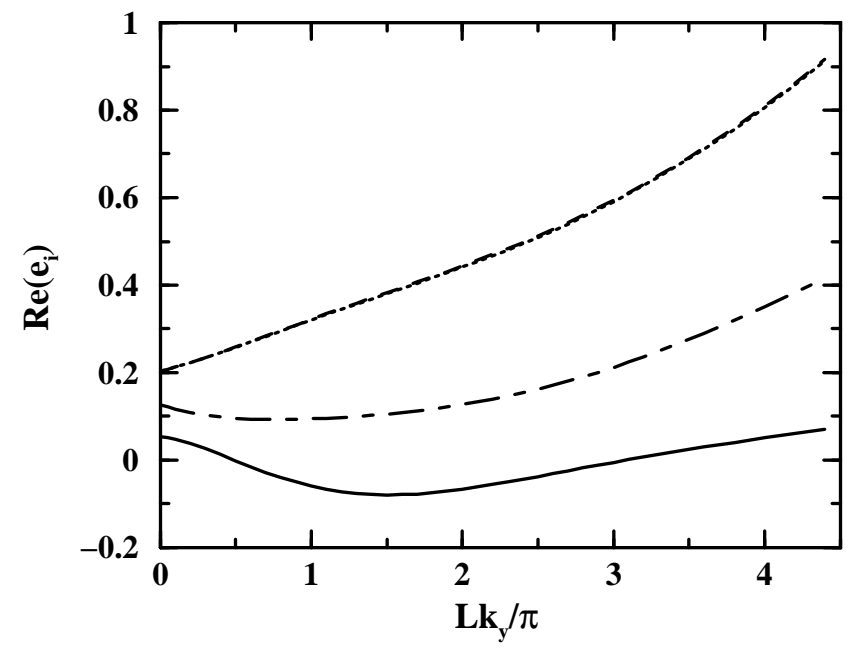

FIG. 5 


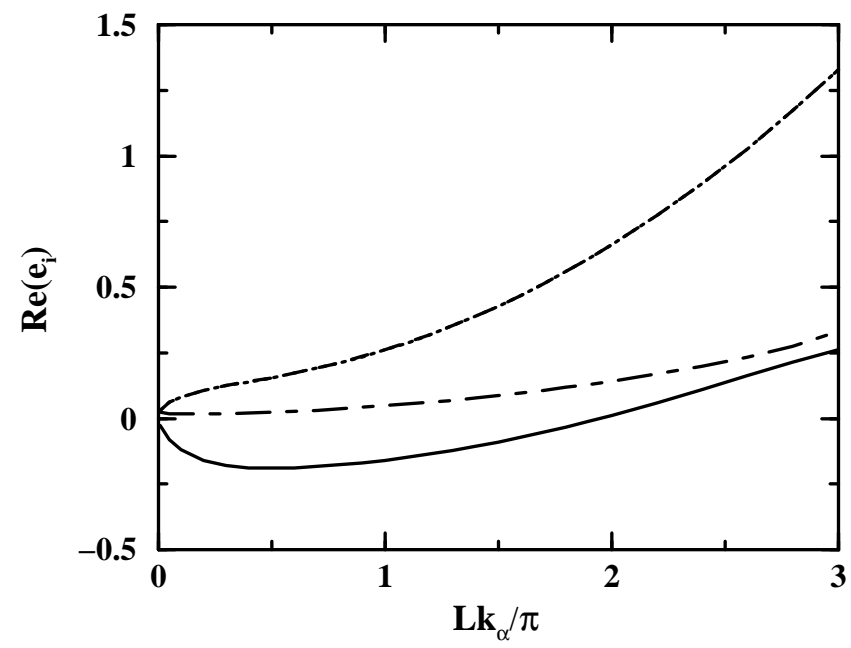

FIG. 6 


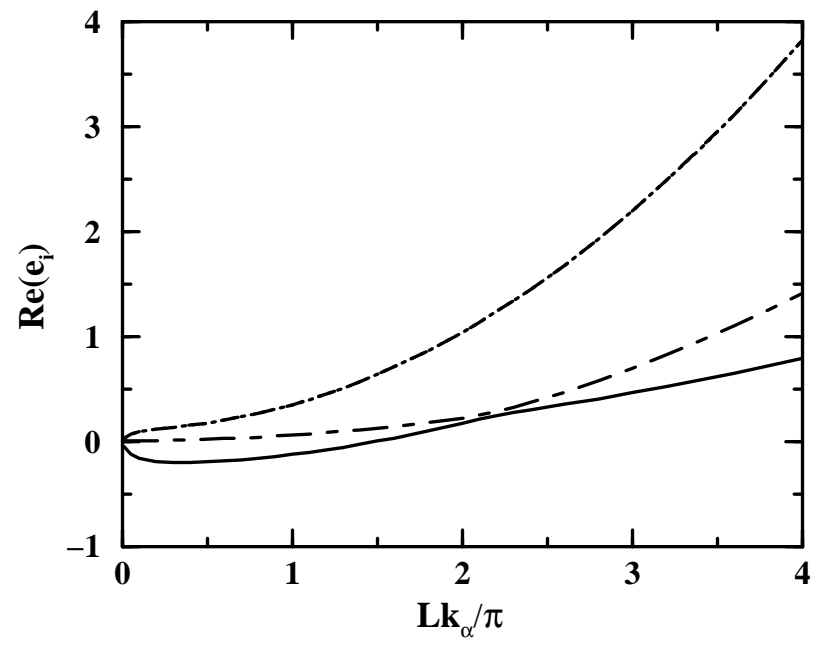

FIG. 7 


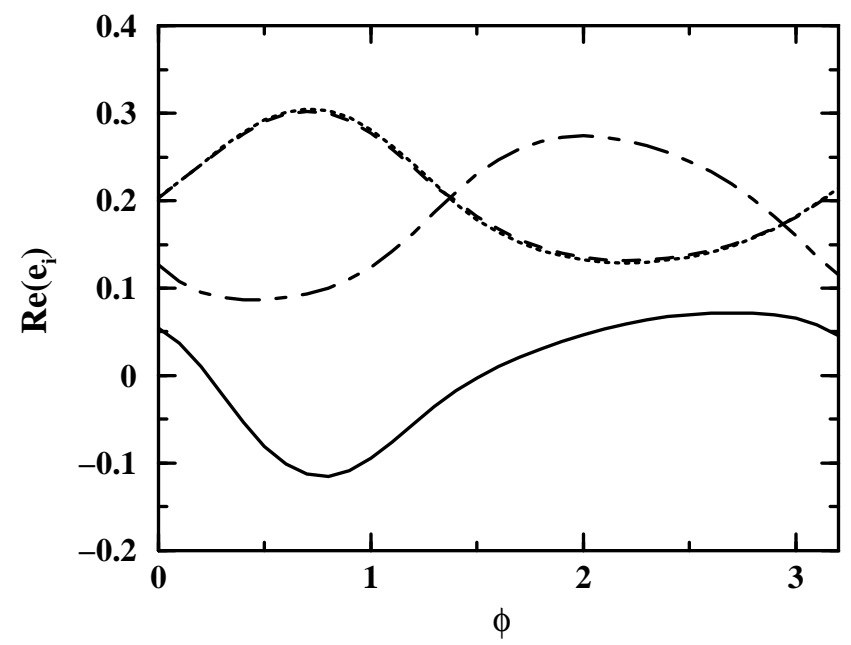

FIG. 8 


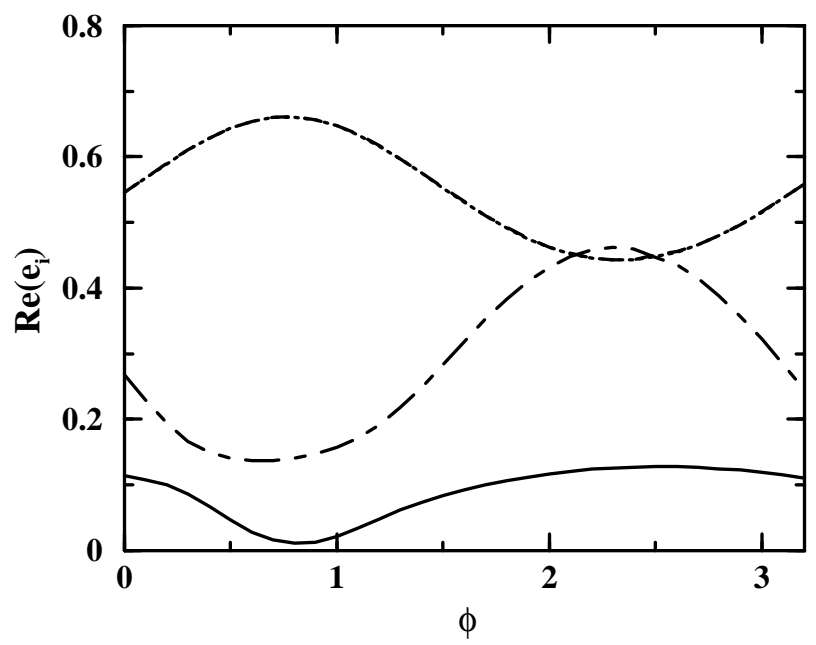

FIG. 9 


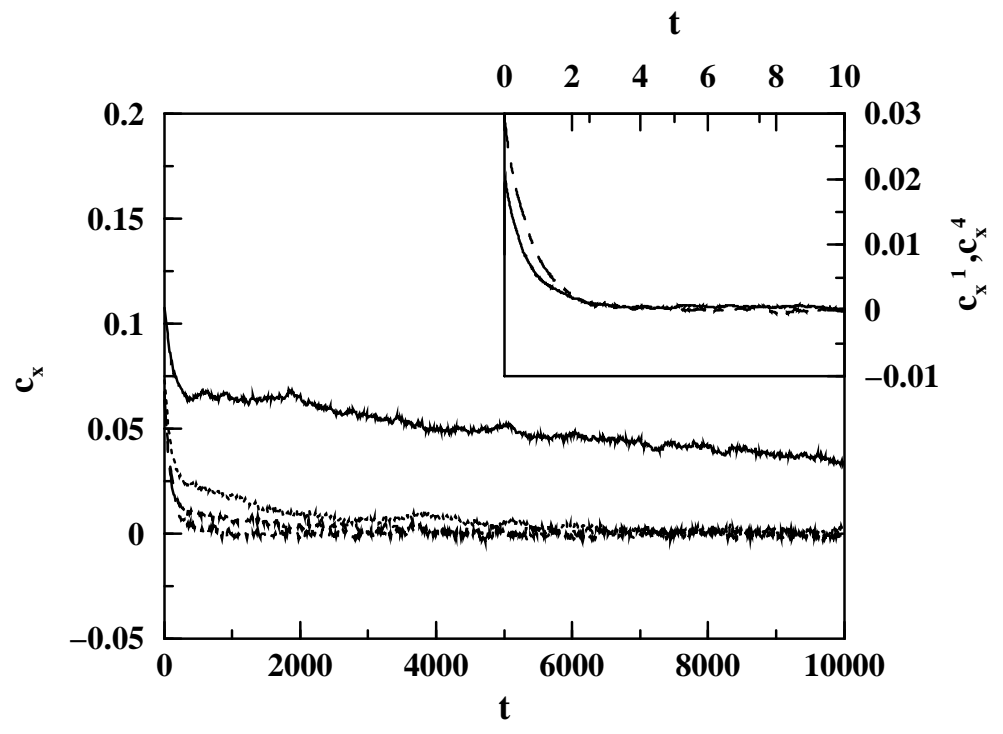

FIG. 10 


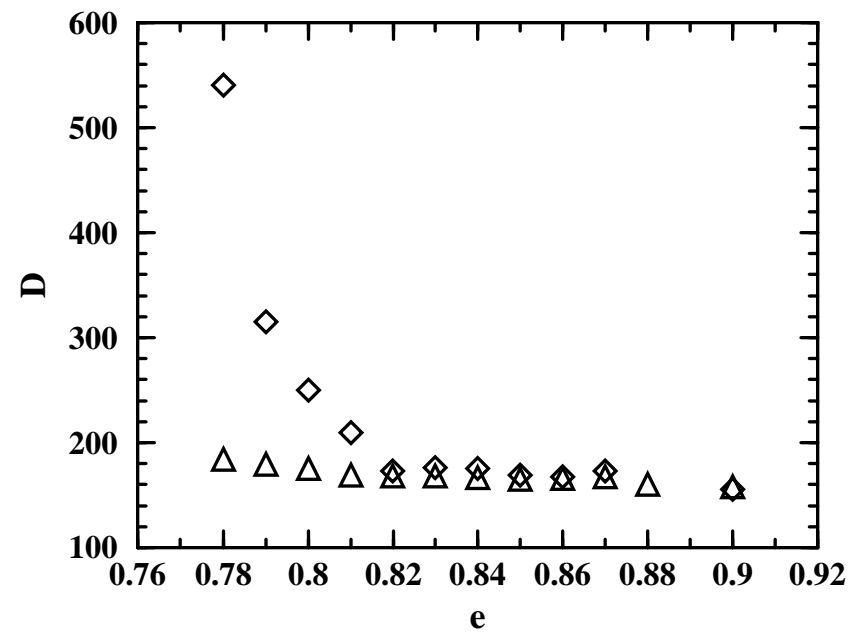

FIG. 11 